\title{
Proof by rotation
}

\author{
Paul Levrie
}

The first proof by rotation I ever saw was probably this proof that $1 / \infty$ is equal to zero, starting from $1 / 0=\infty$ :

$$
\frac{1}{0}=\infty \Rightarrow-10=8 \Rightarrow-18=0 \Rightarrow \frac{1}{\infty}=0
$$

which I found on a blog by Tanya Khovanova [2]. Later, while reading a book about Carl Friedrich Gauss, I started wondering if the young Gauss didn't use rotation to find his trick to calculate the sum of the first 100 numbers. The eight year old Gauss wrote the numbers 1, 2, ., 100 in a row, and then again in the opposite order in the row below. Then he added the columns, each of which totaled 101, from which he easily found the sum. He might have imagined it like this: Write down the numbers next to each other, rotate the result 180 degrees, and merge the original with the rotated version. You get something as in Figure 1 [3].

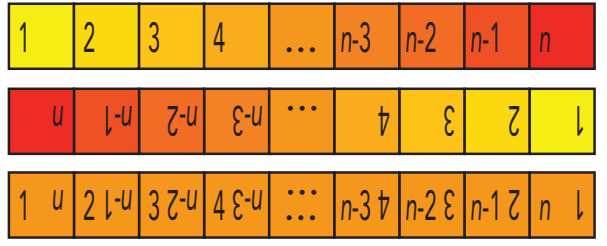

Figure 1: Gauss's trick to calculate the sum of the first $n$ numbers

The numbers in each square have the same sum $n+1$. Hence we find:

$$
2 \cdot(1+2+3+\ldots+n)=n(n+1) \quad \Rightarrow \quad 1+2+3+\ldots+n=\frac{n(n+1)}{2} .
$$

Is generalization of this method possible? Yes it is. We can use it to find the formula for the sum of the first $n$ squares, using an equilateral triangle. We'll do it for $n=4$ (Figure 2). Note that the sum of the entries in the left triangle is equal to $1^{2}+2^{2}+3^{2}+4^{2}$. If we rotate this triangle twice over 120 degrees, and put the results together in one triangle, we get this:
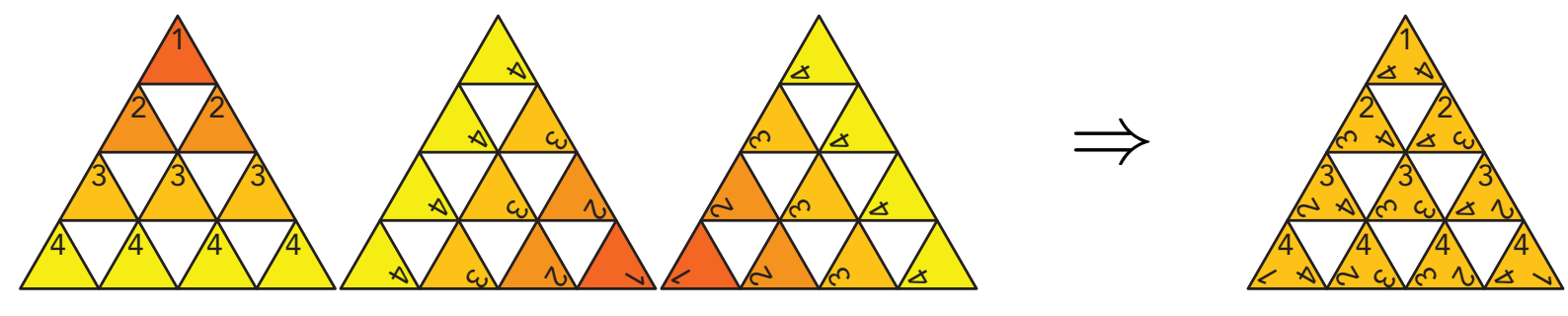

Figure 2: Using rotation to find the sum of the squares of the numbers 1, 2, 3, 4 


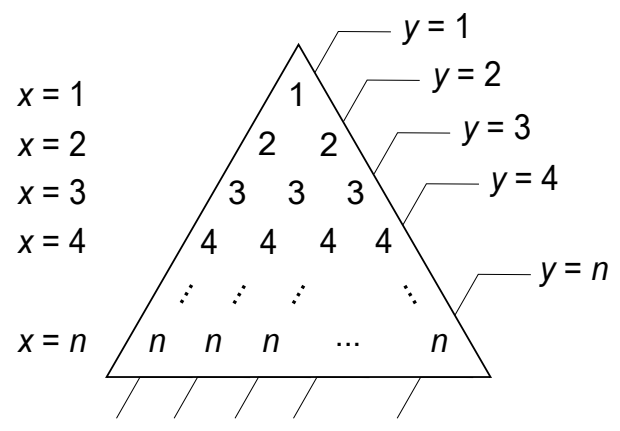

Figure 3: Giving coordinates to every position in the triangle

The sum of the numbers in each little triangle is the same for all triangles. To prove this in the general case, we give every number in the triangle a set of coordinates $(x, y)$, as in Figure 3.

One rotation moves the point with coordinates $(x, y)$ to the new position $(n-x+y, n-x+1)$. Hence, if we look at a particular triangle, for instance the one at position $(x, y)$, after the merging process it will contain the 3 numbers associated with the positions

$$
(x, y),(n-x+y, n-x+1),(n-y+1, x-y+1) .
$$

Since the number in a certain position is precisely its first coordinate in this case, we find for the sum of the numbers in one little triangle:

$$
x+(n-x+y)+(n-y+1)=2 n+1 .
$$

We have 3 copies of the original triangle, hence we may conclude:

$$
3 \cdot\left(1^{2}+2^{2}+3^{2}+\ldots+n^{2}\right)=(1+2+3+\ldots+n) \cdot(2 n+1)
$$

and this can be rewritten using Gauss's result:

$$
1^{2}+2^{2}+3^{2}+\ldots+n^{2}=\frac{n(n+1)(2 n+1)}{6} .
$$

Exercise 1 [4]. Use this method to calculate the sum of the first $n$ triangular numbers.

Exercise 2 [1]. Use a similar method to find the sum of the first $n$ third powers. Hint - Use the following picture:

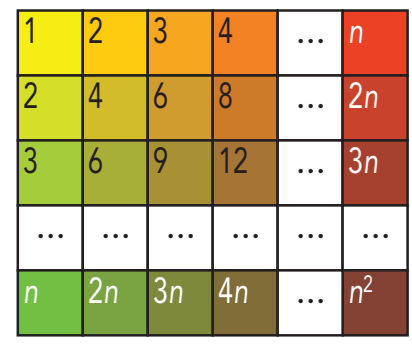

Challenge. Find a similar proof for the sum of the first $n$ fourth powers. (Not yet solved.)

Sources of funding: there are no sources of funding.

Conflict of interest: the author declares that he has no conflict of interest. 


\section{References}

[1] É. Barbier, Démonstration élémentaire du théorème relatif à la somme des cubes des nombres secondaires, Bulletin des sciences mathématiques et astronomiques $2^{e}$ série, 7,1 (1883), 42-43.

[2] http://blog.tanyakhovanova.com/2012/05/a-visual-proof/

[3] P. Levrie, Bewijs door rotatie?, Pythagoras, 53, 2 (2013), 24-25.

[4] J. J. Watkins, Number Theory: A Historical Approach, Princeton University Press, Princeton, New Jersey, 2014.

Faculty of Applied Engineering, UAntwerpen, Salesianenlaan 90, B-2660 Hoboken (Antwerp), Belgium

Department of Computer Science, KU Leuven, Celestijnenlaan 200A, P.O. Box 2402, B-3001 Heverlee (Leuven), Belgium

paul.levrie@cs.kuleuven.be 